\title{
Operational approach to quantum limits on polarization measurement
}

\author{
T. Hakioğlu ${ }^{\mathrm{a}}$, A.S. Shumovsky ${ }^{\mathrm{a}, 1}$, O. Aytür ${ }^{\mathrm{b}}$ \\ a Department of Physics, Bilkent University, TR-06533 Bilkent, Ankara, Turkey \\ b Department of Electrical and Electronics Engineering, Bilkent University, TR-06533 Bilkent, Ankara, Turkey
}

Received 19 July 1994; revised manuscript received 21 September 1994; accepted for publication 21 September 1994

Communicated by B. Fricke

\begin{abstract}
We discuss the polarization measurement of a fully polarized coherent field in the weak intensity quantum limit within the framework of operational approach which was recently discussed by Noh, Fougéres and Mandel. We mainly focus on the fluctuations in the measurement of the parameters of polarization and on the uncertainty relations between them. A dependence of these parameters and their quantum fluctuations on the parameters of the initial coherent field is found.
\end{abstract}

The existence of a well-behaved Hermitian quantum mechanical phase operator is a controversy lasting from the early days of quantum mechanics [1-4]. Most of the literature in this area is mainly concentrated on the mathematical description of the optical quantum phase for the free electromagnetic field. Although these descriptions are equally satisfying for the classical limiting case, they give mutually conflicting results in the cases of extremely low intensities or in strongly non-classical (e.g. squeezed light) distributions [5]. An operational approach to the measurement of the relative quantum phase between two optical fields has been developed recently by Noh, Fougéres and Mandel (NFM) [6,7]. In this formulation, the relative phase is described in terms of the photon counting operators which are defined directly by the measurement.

\footnotetext{
' Also at Bogoliubov Laboratory for Theoretical Physics, Quantum Optics Division, Dubna, Moscow, Russian Federation.
}

In this Letter, we investigate the quantum measurement of the polarization of a coherent optical field using an operational approach. The advantage of this approach is that the quantum fluctuations of the polarization state can be formulated in terms of the quantum statistical fluctuations of the number of detected photons, even in the weak field limit. We first outline a classical polarization measurement scheme and then investigate the quantum mechanical case.

Classically, the state of polarization of a monochromatic optical field, $E_{i}=\epsilon_{i} \cos \left(\omega t+\delta_{i}\right)$, where $i=1,2$ are the indices of two pre-selected orthogonal polarization eigenmodes, can be fully described by the four Stokes parameters $s_{m}(m=0,1,2,3)[8-10]$. Here we only consider the Stokes parametrization of a fully polarized field. For this case

$$
\begin{aligned}
& s_{0}=\frac{1}{2}\left(\left\langle E_{1}^{2}\right\rangle+\left\langle E_{2}^{2}\right\rangle\right), \quad s_{1}=\frac{1}{2}\left(\left\langle E_{1}^{2}\right\rangle-\left\langle E_{2}^{2}\right\rangle\right), \\
& s_{2}=\left(\left\langle E_{1}^{2}\right\rangle\left\langle E_{2}^{2}\right\rangle\right)^{1 / 2} \cos \phi \\
& s_{3}=\left(\left\langle E_{1}^{2}\right\rangle\left\langle E_{2}^{2}\right\rangle\right)^{1 / 2} \sin \phi
\end{aligned}
$$


where $\phi=\delta_{2}-\delta_{1}$ is the optical phase and $\left\langle E_{i}^{2}\right\rangle$ is the intensity of the $i$ th component with the brackets indicating the average over the time interval of the measurement. For an optical field this measurement interval is much greater than the period but it is also smaller than the longitudinal coherence time $[7,11]$. Note that the Stokes parameters are not all independent, but are related by the equality $s_{0}^{2}=s_{1}^{2}+s_{2}^{2}+s_{3}^{2}$.

In the classical case, the $s_{m}$ can be measured using a somewhat similar optical setup to that of NFM, as shown in Fig. 1. The beam whose polarization is to be measured is firstly split into two at the non-polarizing beam splitter $\mathrm{BS}_{1}$. One of the output beams from $\mathrm{BS}_{1}$ is sent to a polarizing beam splitter $\mathrm{PBS}_{1}$, which defines two linearly polarized orthogonal polarization eigenmodes $i=1,2$. Then $I_{1}$ and $I_{2}$ are measured as the output field intensities. The other output beam from $\mathrm{BS}_{1}$ is further split at $\mathrm{BS}_{2}$ with the purpose of measuring $\sin \phi$ and $\cos \phi$ simultaneously. One of the output beams from $\mathrm{BS}_{2}$ is analyzed with $\mathrm{PBS}_{2}$, which is oriented at a $45^{\circ}$ angle with respect to the axes $i=$ 1,2 defined by $\mathrm{PBS}_{1}$. The intensities measured after $\mathrm{PBS}_{2}$ are $I_{3}$ and $I_{4}$. The other output beam from $\mathrm{BS}_{2}$ goes through a quarter-wave $\left(\frac{1}{4} \lambda\right)$ plate whose fast and slow axes are aligned along the $i=1,2$ directions. This beam is then anayzed at $\mathrm{PBS}_{3}$ whose axes are aligned with $\mathrm{PBS}_{2}$. The intensities measured after $\mathrm{PBS}_{3}$ are $I_{5}$ and $I_{6}$.

For $50 \%$ non-polarizing beam splitters, the measured intensities $I_{i}$ are related to the input field by

$$
\begin{aligned}
& I_{1}=\frac{1}{2}\left\langle E_{1}^{2}\right\rangle, \quad I_{2}=\frac{1}{2}\left\langle E_{2}^{2}\right\rangle, \\
& I_{3}=\frac{1}{4}\left(\left\langle E_{1}^{2}\right\rangle+\left\langle E_{2}^{2}\right\rangle+2 \sqrt{\left\langle E_{1}^{2}\right\rangle\left\langle E_{2}^{2}\right\rangle} \cos \phi\right), \\
& I_{4}=\frac{1}{4}\left(\left\langle E_{1}^{2}\right\rangle+\left\langle E_{2}^{2}\right\rangle-2 \sqrt{\left\langle E_{1}^{2}\right\rangle\left\langle E_{2}^{2}\right\rangle} \cos \phi\right), \\
& I_{5}=\frac{1}{4}\left(\left\langle E_{1}^{2}\right\rangle+\left\langle E_{2}^{2}\right\rangle+2 \sqrt{\left\langle E_{1}^{2}\right\rangle\left\langle E_{2}^{2}\right\rangle} \sin \phi\right), \\
& I_{6}=\frac{1}{4}\left(\left\langle E_{1}^{2}\right\rangle+\left\langle E_{2}^{2}\right\rangle-2 \sqrt{\left\langle E_{1}^{2}\right\rangle\left\langle E_{2}^{2}\right\rangle} \sin \phi\right) .
\end{aligned}
$$

From these relations the classical Stokes parameters can be written as

$s_{0}=I_{1}+I_{2}, \quad s_{1}=I_{1}-I_{2}$,

$s_{2}=I_{3}-I_{4}, \quad s_{3}=I_{5}-I_{6}$.

The $s_{m}$ defined by Eqs. (2) and (3) depend on the initial choice of the orthogonal axes 1,2 . The polar- ization state, hence the orientation of the polarization ellipse, for any input field will be measured relative to these axes. We are interested in the fluctuations associated with the measurement of the polarization state. Such fluctuations are very important when the measured field is very weak or in some quantum state. In relation with the measurement of these fluctuations we first define the classical angular functions,

$\sin \theta=2 \frac{\left(I_{1} I_{2}\right)^{1 / 2}}{I_{1}+I_{2}}, \quad \cos \theta=\frac{I_{1}-I_{2}}{I_{1}+I_{2}}$,

$\cos \phi=\frac{I_{3}-I_{4}}{\left[\left(I_{3}-I_{4}\right)^{2}+\left(I_{5}-I_{6}\right)^{2}\right]^{1 / 2}}$,

$\sin \phi=\frac{I_{5}-I_{6}}{\left[\left(I_{3}-I_{4}\right)^{2}+\left(I_{5}-I_{6}\right)^{2}\right]^{1 / 2}}$.

Using Eq. (4), Eq. (3) can be described in a polar decomposition. If the axes 1,2 are the principle axes of the ellipse of polarization, then $\cos \theta$ is related to the ellipticity angle, and $\phi=\delta_{2}-\delta_{1}$ becomes the optical phase between the major and minor axis components [8]. Although $\theta$ and $\phi$ can be mixed by rotating the frame in which they are defined, they are closely connected with the geometry of the ellipse.

The setup in Fig. 1 allows us to make measurements on $\theta$ and $\phi$ related to the polarization state without employing any interference. In the laboratory, it is technically difficult to eliminate the influence of the random path lenght fluctuations on the measurement of the optical phase. It should be emphasized that in our proposed setup, each component goes through the same optical path in both the $\cos \phi$ and the $\sin \phi$ measurements. There is also no restriction on the arm lenghts $\mathrm{BS}_{2}-\mathrm{PBS}_{3}$ and $\mathrm{BS}_{2}-\mathrm{PBS}_{2}$ imposed by the temporal coherence length. Therefore, the setup in Fig. 1 is immune to random phase fluctuations resulting from mechanical vibrations of various optical components.

Having defined our measurement, we next look at the quantum description of the polarization state. Quantum meachanically, the unused ports in the beam splitters are considered as input for vacuum fields. Therefore, we include in Fig. 1 the vacuum fields $v^{(1)}, v^{(2)}$ for $\mathrm{BS}_{1}$ and $\mathrm{BS}_{2}$ and $w^{(1)}, w^{(2)}, w^{(3)}$ for $\mathrm{PBS}_{1}, \mathrm{PBS}_{2}$ and $\mathrm{PBS}_{3}$, respectively.

The annihilation operators associated with the optical fields at the output arms of $\mathrm{PBS}_{1}$ are related to those at the input through 


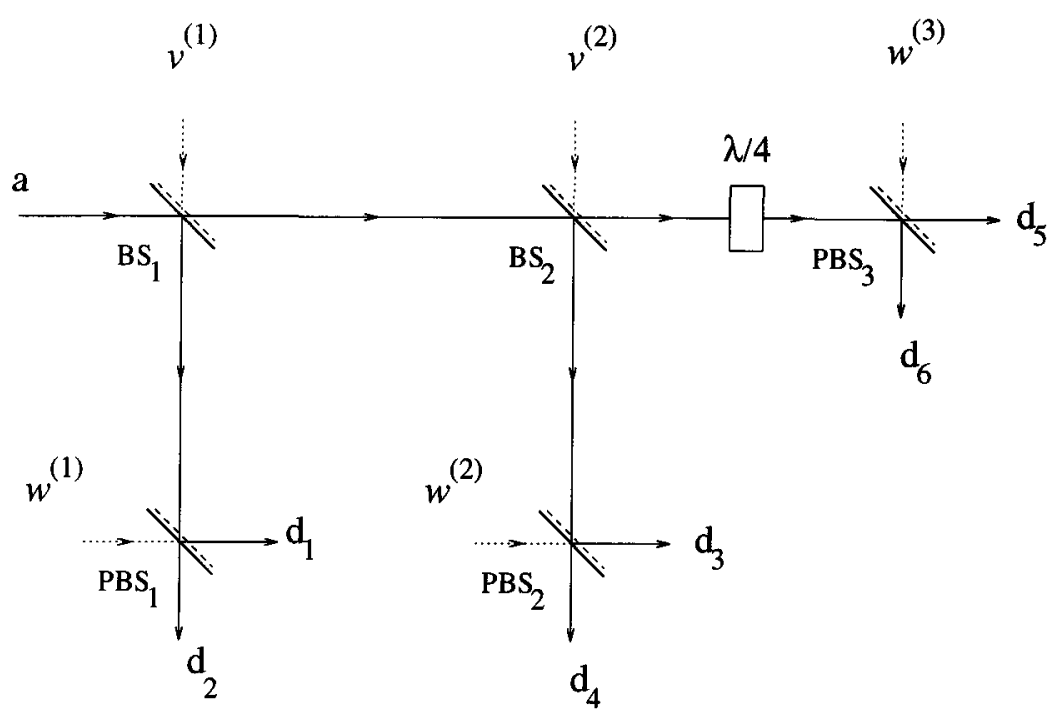

Fig. 1. Experimental setup for the measurement of all Stokes parameters. Note that this setup can be used in the measurement of a partially polarized field as well. However, here we only concentrate on the fully polarized case.

$\hat{d}_{1}=r \hat{a}_{1}+t \hat{v}_{1}^{(1)}, \quad \hat{d}_{2}=r \hat{a}_{2}+t \hat{v}_{2}^{(1)}$,

where $r=\mathrm{i} / \sqrt{2}$ and $t=1 / \sqrt{2}$ are the field reflection and transmission coefficients, and $\hat{v}_{j}^{(1)}(j=1,2)$ describes the polarized vacuum field in the $j$ direction entering through the vacuum port of $\mathrm{BS}_{1}$. The vacuum fields $\hat{w}^{(k)}(k=1,2,3)$ do not couple with the measured field operators since they are orthogonal to them. Therefore, we do not include any of these orthogonal vacuum field components, as they do not contribute to the fluctuations of the intensity in our operational formalism. We next define the operators

$\hat{S}_{\theta}=2 \frac{\left(\hat{n}_{1} \hat{n}_{2}\right)^{1 / 2}}{\hat{n}_{1}+\hat{n}_{2}}, \quad \hat{C}_{\theta}=\frac{\hat{n}_{1}-\hat{n}_{2}}{\hat{n}_{1}+\hat{n}_{2}}$,

where $\hat{n}_{j}=\hat{d}_{j}^{\dagger} \hat{d}_{j}, j=1,2$, are the photon number operators for the fields measured at the detectors 1 , 2 . Assuming ideal detectors with unit quantum efficiency, the measured value of $\hat{n}_{j}$ represents the photocurrents at the detectors. Since $\left[\hat{v}_{1}^{(1)}, \hat{v}_{2}^{(1) \dagger}\right]=0$ and $\left[\hat{a}_{1}, \hat{a}_{2}^{\dagger}\right]=0$ due to independence, we have $\left[\hat{d}_{1}, \hat{d}_{2}^{\dagger}\right]=$ 0 . This relation together with the use of the semiclassical analogy justifies the definition of the operators in Eq. (6). As a consequence of $\left[\hat{d}_{1}, \hat{d}_{2}^{\dagger}\right]=0$, we also have $\left[\hat{S}_{\theta}, \hat{C}_{\theta}\right]=0$ and $\hat{S}_{\theta}^{2}+\hat{C}_{\theta}^{2}=1$.
On the other hand, at the output ports of $\mathrm{PBS}_{2}$ and $\mathrm{PBS}_{3}$, the field operators $\hat{d}_{3}, \hat{d}_{4}, \hat{d}_{5}, \hat{d}_{6}$ are

$$
\begin{aligned}
\hat{d}_{3} & =\frac{1}{\sqrt{2}}\left[\left(t r \hat{a}_{1}+r^{2} \hat{v}_{1}^{(1)}+t \hat{v}_{1}^{(2)}\right)\right. \\
& \left.+\left(t r \hat{a}_{2}+r^{2} \hat{v}_{2}^{(1)}+t \hat{v}_{2}^{(2)}\right)\right], \\
\hat{d}_{4} & =\frac{1}{\sqrt{2}}\left[-\left(t r \hat{a}_{1}+r^{2} \hat{v}_{1}^{(1)}+t \hat{v}_{1}^{(2)}\right)\right. \\
& \left.+\left(t r \hat{a}_{2}+r^{2} \hat{v}_{2}^{(1)}+t \hat{v}_{2}^{(2)}\right)\right], \\
\hat{d}_{5} & =\frac{1}{\sqrt{2}}\left[\mathrm{i}\left(t^{2} \hat{a}_{1}+\operatorname{tr} \hat{v}_{1}^{(1)}+r \hat{v}_{1}^{(2)}\right)\right. \\
& \left.+\left(t^{2} \hat{a}_{2}+\operatorname{tr} \hat{v}_{2}^{(1)}+r \hat{v}_{2}^{(2)}\right)\right], \\
\hat{d}_{6} & =\frac{1}{\sqrt{2}}\left[-\mathrm{i}\left(t^{2} \hat{a}_{1}+t r \hat{v}_{1}^{(1)}+r \hat{v}_{1}^{(2)}\right)\right. \\
& \left.+\left(t^{2} \hat{a}_{2}+t r \hat{v}_{2}^{(1)}+r \hat{v}_{2}^{(2)}\right)\right] .
\end{aligned}
$$

Here $\hat{v}_{j}^{(2)}(j=1,2)$ describes the polarized vacuum field in the $j$ direction through the vacuum port of $\mathrm{BS}_{2}$. Then, by the same analogy with Eq. (4) we have the following relations for the optical phase operators,

$$
\begin{aligned}
& \hat{C}_{\phi}=\frac{\hat{n}_{3}-\hat{n}_{4}}{\left[\left(\hat{n}_{3}-\hat{n}_{4}\right)^{2}+\left(\hat{n}_{5}-\hat{n}_{6}\right)^{2}\right]^{1 / 2}}, \\
& \hat{S}_{\phi}=\frac{\hat{n}_{5}-\hat{n}_{6}}{\left[\left(\hat{n}_{3}-\hat{n}_{4}\right)^{2}+\left(\hat{n}_{5}-\hat{n}_{6}\right)^{2}\right]^{1 / 2}}
\end{aligned}
$$


with $\hat{n}_{j}=\hat{d}_{j}^{\dagger} \hat{d}_{j}(j=3,4,5,6)$. Here $\hat{n}_{3}-\hat{n}_{4}$ and $\hat{n}_{5}-\hat{n}_{6}$ are

$$
\begin{gathered}
\hat{n}_{3}-\hat{n}_{4}=\left[\left(r^{*} t^{*} \hat{a}_{2}^{\dagger}+r^{*} r^{*} \hat{v}_{2}^{(1)}+t^{*} \hat{v}_{2}^{(2) \dagger}\right)\right. \\
\left.\times\left(r t \hat{a}_{1}+r^{2} \hat{v}_{1}^{(1)}+t \hat{v}_{1}^{(2)}\right)+\text { h.c. }\right], \\
\hat{n}_{5}-\hat{n}_{6}=\mathrm{i}\left[\left(t^{*^{2}} \hat{a}_{2}^{\dagger}+t^{*} r^{*} \hat{v}_{2}^{(1)}+r^{*} \hat{v}_{2}^{(2) \dagger}\right)\right. \\
\left.\quad \times\left(t^{2} \hat{a}_{1}+t r \hat{v}_{1}^{(1)}+r \hat{v}_{1}^{(2)}\right)-\text { h.c. }\right],
\end{gathered}
$$

where h.c. denotes Hermitian conjugate. Using Eqs. (7) it can be shown that neither $\hat{n}_{5}$ nor $\hat{n}_{6}$ commute with $\hat{n}_{4}$ or $\hat{n}_{3}$. Here $\left[\hat{C}_{\phi}, \hat{S}_{\phi}\right]=0$ follows explicitly from the existence of the vacuum fields in the combination $\hat{n}_{3}-\hat{n}_{4}$ and $\hat{n}_{5}-\hat{n}_{6}$. On the other hand, for the commutation $\left[\hat{S}_{\theta}, \hat{C}_{\theta}\right]=0$ of the previous case, the vacuum field $\hat{v}^{(1)}$ does not play any role. This is because at $\mathrm{PBS}_{1}$ the orthogonal field operators $\hat{d}_{1}$ and $\hat{d}_{2}$ do not have the same components of the vacuum field $\hat{v}^{(1)}$.

We first analyze the fluctuation

$$
D_{\theta}=\sqrt{\left(\Delta S_{\theta}\right)^{2}+\left(\Delta C_{\theta}\right)^{2}}
$$

in the measurement of $\hat{S}_{\theta}$ and $\hat{C}_{\theta}$ where the variances are $\left(\Delta S_{\theta}\right)^{2}=\left\langle\left(\hat{S}_{\theta}-\left\langle S_{\theta}\right\rangle\right)^{2}\right\rangle$ and $\left(\Delta C_{\theta}\right)^{2}=\left\langle\left(\hat{C}_{\theta}-\right.\right.$ $\left.\left.\left\langle C_{\theta}\right\rangle\right)^{2}\right\rangle$, respectively. The brackets indicate quantum averages calculated with respect to the initial state $\left|\psi_{\mathrm{IN}}\right\rangle$. In this semiclassical operational approach, the measured field is treated classically, and the joint probability distribution of the detectors is treated quantum mechanically $[11,12]$. At $\mathrm{PBS}_{1}$ the measured value of a general operator $f\left(\hat{n}_{1}, \hat{n}_{2}\right)$ is given by [7]

$$
\left\langle\hat{f}\left(\hat{n}_{1}, \hat{n}_{2}\right)\right\rangle=\mathcal{N} \sum_{n_{1}, n_{2}}^{\prime} f\left(n_{1}, n_{2}\right)\left\langle\mathcal{P}\left(\hat{n}_{1}, \hat{n}_{2}\right)\right\rangle,
$$

where $\mathcal{N}$ is the normalization, and $\left\langle\mathcal{P}\left(\hat{n}_{1}, \hat{n}_{2}\right)\right\rangle=$ $\left\langle\psi_{\mathrm{IN}}\left|\mathcal{P}\left(\hat{n}_{1}, \hat{n}_{2}\right)\right| \psi_{\mathrm{IN}}\right\rangle$, where $\left|\psi_{\mathrm{IN}}\right\rangle$ is the initial photon state as observed in $\mathrm{PBS}_{1}$. Here $\mathcal{P}\left(\hat{n}_{1}, \hat{n}_{2}\right)$ is given by the normal ordered product

$\mathcal{P}\left(\hat{n}_{1}, \hat{n}_{2}\right)=\prod_{j=1}^{2}:\left(\hat{d}_{j}^{\dagger} \hat{d}_{j}\right)^{n_{j}} \exp \left(-\hat{d}_{j}^{\dagger} \hat{d}_{j}\right) / n_{j} !:$,

which is the joint probability distribution of detecting $n_{j}$ photons at detectors 1,2 . To calculate the fluctuation $\Delta f$ in the measurement of the operator $\hat{f}$, we find $\langle\hat{f}\rangle$ and $\left\langle\hat{f}^{2}\right\rangle$ using Eq. (10). An important point is that there is an ill-defined contribution of the unobserved data corresponding to $n_{1}=n_{2}=0$ in the calculation of $D_{\theta}$. The relative contributions of these unobserved events in the total average is not small if the initial average field intensities are weak (i.e. $\bar{n}_{1}, \bar{n}_{2}<1$ ). Therefore, we discard these data points by subtracting the probability $\langle\mathcal{P}(0,0)\rangle$ from the normalization and also excluding the corresponding terms in the summation (indicated by the prime). Hence the normalization, in the operational sense, is given by $\mathcal{N}^{-1}=$ $1-\langle\mathcal{P}(0,0)\rangle[7]$.

According to the measurement scheme in Fig. 1 the orientation of the first polarizing beam splitter $\mathrm{PBS}_{1}$ is arbitrary. Thus, fluctuations should not only be examined with respect to the independent average number of particles $\bar{n}_{1}$ and $\bar{n}_{2}$ but also with respect to their ratio $\eta=\bar{n}_{2} / \bar{n}_{1}$. Let us assume that the initial beam is given in a product coherent state with average number of particles $|\alpha|^{2}$ and $|\beta|^{2}$ with respect to some fixed reference axes $1^{0}$ and $2^{0}$. Then $\left|\left\langle n_{1} n_{2} \mid \psi_{\mathrm{IN}}\right\rangle\right|^{2}=$ $P\left(n_{1},|\alpha|^{2}\right) \otimes P\left(n_{2},|\beta|^{2}\right)$ where $P$ describes the number probability distribution of a coherent state with mean photon numbers $|\alpha|^{2}$ and $|\beta|^{2}$. If $\alpha=|\alpha| \mathrm{e}^{\mathrm{i} \delta_{\alpha}}$ and $\beta=|\beta| \mathrm{e}^{\mathrm{i} \delta_{\beta}}$ one also has a coherent distribution in 1,2 frame with average number of particles,

$\bar{n}_{1}=\frac{1}{2}|\alpha \cos \gamma-\beta \sin \gamma|^{2}$,

$\bar{n}_{2}=\frac{1}{2}|\beta \cos \gamma+\alpha \sin \gamma|^{2}$,

with $\gamma$ describing the arbitrary angle between the frames $1^{0}, 2^{0}$ and 1,2 , and the overall $\frac{1}{2}$ is due to the BS $_{1}$. In Fig. $2 D_{\theta}$ is shown as a function of $\bar{n}_{1}$ for $\eta=0.1,1,10$. The fluctuation increases rapidly to its maximum value from the classically expected value of zero as the average number of detected photons decreases. The largest fluctuation is associated with $\eta=1$. Even if $|\alpha| \neq|\beta| \eta$ can still be very close to one if the axes of $\mathrm{PBS}_{1}$ are oriented relative to $1^{0}, 2^{0}$ by certain amount which is determined by Eq. (12).

We now study $D_{\phi}=\left[\left(\Delta \hat{S}_{\phi}\right)^{2}+\left(\Delta \hat{C}_{\phi}\right)^{2}\right]^{1 / 2}$ for $\hat{C}_{\phi}$ and $\hat{S}_{\phi}$ operators. Here the operator $\hat{f}\left(\hat{n}_{3}, \hat{n}_{4}, \hat{n}_{5}, \hat{n}_{6}\right)$ in Eq. (10) is given by the corresponding $\hat{C}_{\phi}$ and $\hat{S}_{\phi}$ operators defined by Eq. (8), and $\mathcal{P}\left(\hat{n}_{1}, \hat{n}_{2}\right)$ is replaced by $\mathcal{P}\left(\hat{n}_{3}, \hat{n}_{4}, \hat{n}_{5}, \hat{n}_{6}\right)$. The calculated $D_{\phi}$ is shown in Fig. 3. Figs. 2 and 3 indicate that for $\eta=1$, $D_{\theta}$ takes its largest and $D_{\phi}$ its smallest values compared to other possible values of $\eta$ for small values of $\bar{n}_{1}$. As $\bar{n}_{1}$ gets large enough, the roles of $D_{\theta}$ and $D_{\phi}$ 


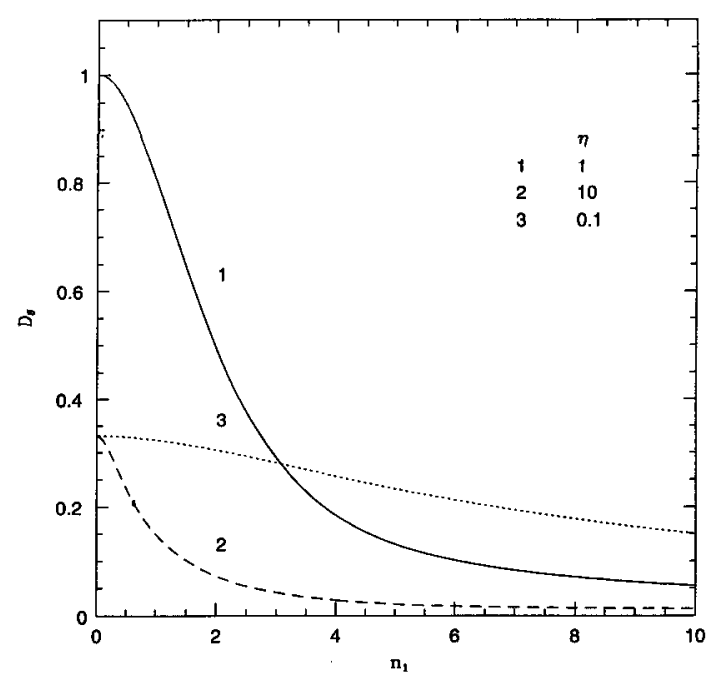

Fig. 2. Fluctuation in $\theta$ as a function of the average number of particles measured at the detector $d_{1}$.

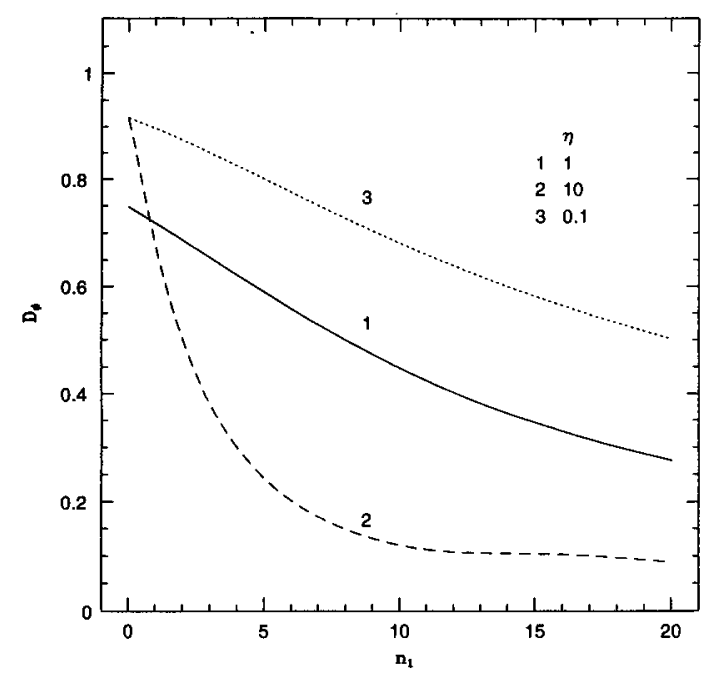

Fig. 3. Fluctuation in $\phi$ as a function of the average number of particles measured at the detector $d_{2}$.

interchange. We will now briefly study this behaviour in connection with an uncertainty principle.

The analysis of fluctuations in the polarization measurement of weak fields yields interesting results. Figs. 2 and 3 indicate that the quantum statistics of the initial field has an important influence on the measured parameters of polarization. Although the choice of the axes on which one measures the polarization is arbi-

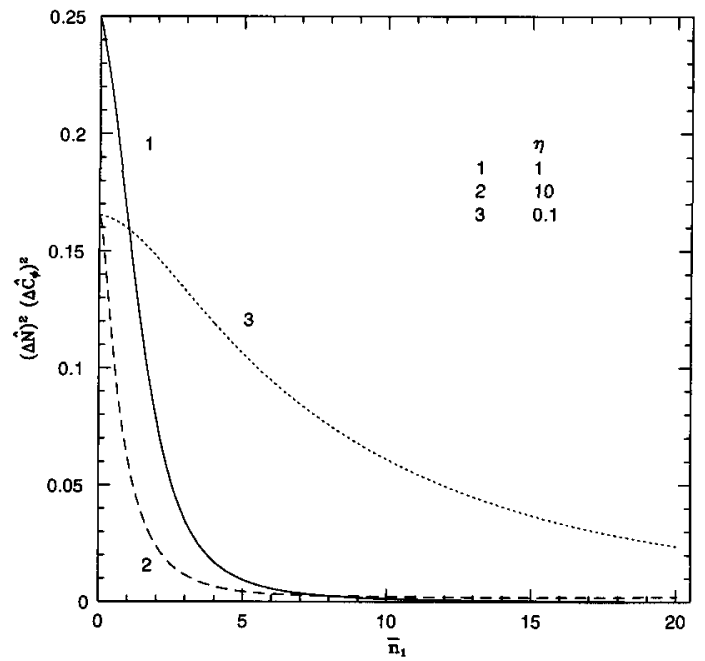

Fig. 4. Uncertainty product described by the I.h.s. of Eq. (13) for $\eta=0.1,1,10$.

trary, fluctuations strongly depend on the relative intensity of the field components measured along the axes. Theoretically, the optical phase $\phi$ is the canonically conjugated operator to the number difference operator $\hat{N}=\hat{n}_{2}-\hat{n}_{1}[1,2]$. Then from Eq. (6) we have $\hat{N}=-\hat{C}_{\theta}\left(\hat{n}_{1}+\hat{n}_{2}\right)$. Hence we expect the fluctuations in $\theta$ - and $\phi$-dependent operators to be regulated by the uncertainty relations

$$
\begin{aligned}
& (\Delta \hat{N})^{2}\left(\Delta \hat{C}_{\phi}\right)^{2} \geqslant \frac{1}{4}\left\langle\hat{S}_{\phi}\right\rangle^{2}, \\
& (\Delta \hat{N})^{2}\left(\Delta \hat{S}_{\phi}\right)^{2} \geqslant \frac{1}{4}\left\langle\hat{C}_{\phi}\right\rangle^{2} .
\end{aligned}
$$

In Fig. 4 the first one of Eqs. (13) is plotted as a function of $\bar{n}_{1}$ for $\eta=0.1,1,10$. We examined it and observed that the unequality is respected for all values of $\bar{n}_{1}$ even though one would expect it to be questionable for $\bar{n}_{1}<1$ due to the subtracted statistics in the averages. For small values of $\bar{n}_{1}, \Delta \hat{N}$ is small due to the number coherent state but does not go to zero like in the pure coherent state. This is also a result of the subtracted, unobservable data. Also in Fig. 4, an interesting feature of Eqs. (13) with respect to $\eta$ deserves some attention. Curves corresponding to two different $\eta$ 's cross each other at $\bar{n}_{1}$ values fixed by the ratio of those $\eta$ 's. For instance, in the range $\bar{n}_{1}<1$ $\eta=1$ yields the largest uncertainty among all possible $\eta$ values. At $\bar{n}_{1} \simeq 1$ a crossover is observed between the curves corresponding to $\eta=0.1$ and $\eta=1$. The 
$\eta=1$ and $\eta=10$ curves cross each other at $\bar{n}_{1} \sim 10$ and then $\eta=1$ yields the smallest uncertainty of all three curves for $\bar{n}_{1}>10$. If, instead of Fig. 4, we had plotted $\left(\Delta \hat{C}_{\theta}\right)^{2}\left(\Delta \hat{C}_{\phi}\right)^{2}$ against $\bar{n}_{1}$ we would have seen the same crossing pattern at approximately the same $\bar{n}_{1}$ values. Naturally because of the form of Eqs. (4), (6) and (8) the point where the crossover is observed scales with respect to $\eta^{2}$. Thus, although $\eta=1$ corresponds to the largest uncertainty in the weak field limit, it is the fastest decaying one with increasing average number of particles.

In conclusion, we believe that the relative intensity dependence of the uncertainty relations (13) described in Fig. 4 is of experimental importance for the measurement of the polarization of weak fields. These theoretically observed features can be easily examined in an experiment described in Fig. 1. There have been a number of studies on the measurement of the probability distribution of the moments of the phase operator [13]. It can be shown that in polarization measurement the widths of the corresponding probability distributions $P_{\theta}$ and $P_{\phi}$ of $\theta$ and $\phi$ dependent operators are correlated in the reciprocal sense due to the uncertainity relations (13). A more detailed analysis of this manifestation of the uncertainty in weak polarization measurements will be given in another publication.

T.H. appreciates financial support by Professor P. Carruthers on his visit in the summer of 1993 to The University of Arizona, where this work was first discussed.

\section{References}

[1] P.A.M. Dirac, Proc. R. Soc. A 114 (1927) 243

[2] P. Carruthers and M.M. Nieto, Phys. Rev. Lett. 14 (1965) 387; Rev. Mod. Phys. 40 (1968) 411;

L. Susskind and J. Glogower, Physics 1 (1964) 49.

[3] D.T. Pegg and S.M. Barnett, Phys. Rev. A 39 (1989) 1665; Phys. Rev. A 43 (1991) 2579.

[4] D. Ellinas, J. Math. Phys. 32 (1991) 135.

[5] J.A. Vaccaro and D.T. Pegg, Opt. Commun. 70 (1989) 529; B.C. Sanders, S.M. Barnett and P.L. Knight, Opt. Commun. 58 (1986) 290.

[6] J.W. Noh, A. Fougéres and L. Mandel, Phys. Rev. Lett. 67 (1991) 1426.

[7] J.W. Noh, A. Fougéres and L. Mandel, Phys. Rev. A 45 (1992) 424; 46 (1992) 2840; E. Wolf, Nuovo Cimento 12 (1954) 884.

[8] R.M. Azzam, Ellipsometry and polarized light (Elsevier, Amsterdam, 1989).

[9] G.G. Stokes, Trans. Cambridge Philos. Soc. 9 (1852) 399.

[10] W.H. McMaster, Am. J. Phys. 22 (1954) 351.

[11] L. Mandel, J. Opt. Soc. Am. 51 (1961) 797; L. Mandel and E. Wolf, Rev. Mod. Phys. 37 (1965) 231.

[12] L. Mandel, Proc. Phys. Soc. 74 (1959) 233; R. Glauber, in: Quantum optics and electronics, eds. C. De Witt, A. Blandin and C. Cohen-Tannoudji (Gordon and Breach, London, 1968).

[13] M. Freyberger and W. Schleich, Phys. Rev. A (1993) R30; J.W. Noh, A. Fougéres and L. Mandel, Phys. Rev. Lett. 71 (1993) 2579.

\footnotetext{
${ }^{2}$ Small shifts of the crossover points from their fixed value determined by $\eta$ 's is due to the truncation errors in the calculation of otherwise infinite summation in Eq. (10).
} 